\title{
Effectiveness of Intraoperative Parathyroid Monitoring (IoPTH) During Minimally-Invasive Parathyroidectomy: A Decade Experience in a Single Center
}

\author{
Dobrinja $C^{1 *}$, Lanzaro A1, Cubisino A ${ }^{1}$, Stenner $E^{2}$, Ruscio $\mathbf{M}^{2}$, Dore $F^{3}$, Bortul $\mathbf{M}^{\mathbf{1}}$ and De Manzini Nicolò ${ }^{1}$ \\ ${ }^{1}$ Division of General Surgery, Department of Medical, Surgical and Health Sciences, Cattinara Teaching Hospital, Strada di Fiume, 34149, Trieste, Italy \\ ${ }^{2}$ Division of Laboratory Medicine, Cattinara Teaching Hospital, Strada di Fiume, 34149, Trieste, Italy \\ ${ }^{3}$ Department of Nuclear Medicine, Cattinara Teaching Hospital, Strada di Fiume, 34149, Trieste, Italy
}

\begin{abstract}
Background: Aim of this study was to analyse our results of oriented parathyroidectomy (minimally-invasive parathyroidectomy (MIP) minimally-invasive video-assisted parathyroidectomy (MIVAP); and to confirm the usefulness of intra-operative parathyroid hormone (PTH) monitoring (ioPTH) when using minimally invasive techniques for treatment of sporadic primary hyperparathyroidism (pHTP).

Material and methods: From June 2005 to May 2017, in the Division of General Surgery of University of Trieste, we operated 137 patients with pHPT. Minimally invasive video-assisted parathyroidectomy (MIVAP) by an anterior approach was proposed for $60(43.8 \%)$ patients with sporadic pHPT and one unequivocally enlarged parathyroid gland on pre-operative ultrasound and $99 \mathrm{mTC}-$ SestaMIBI scintigraphy without previous neck surgery. Intraoperatively, a quick parathyroid assay was used during the last $107(78.1 \%)$ surgical procedures. All patients underwent preoperative investigations of calcemia, phoshoremia and PTH levels and vocal cord function. Age, gender, operative times, pathologic findings, calcemia, PTH, length of hospital stay and complications were retrospectively analyzed.

Results: MIVAP was successfully accomplished in 60 patients. Mean operative time was 87 minutes. Postoperative complications included 12/137 (8.75\%) transient hypocalcemia. No laryngeal nerve palsies, no definitive hypocalcemias, no persistent pHPT and no recurrent pHPT were observed. Pre-operative 99mTc-SestaMIBI scintigraphy revealed the correct position of parathyroid adenoma in 91 cases $(66.4 \%)$

Conclusion: Our decade experience in a single center demonstrated that minimally invasive parathyroidectomy for localized single-gland adenoma seems to have significant advantages, especially in terms of cosmetic results and post-operative pain, if performed by dedicated team, with an adequate activity volume. Moreover, the ioPTH determinations are very important during minimally invasive parathyroidectomy, as it allows avoiding bilateral neck exploration.
\end{abstract}

Keywords: Minimally invasive parathyroidectomy; Intraoperative parathyroid hormone monitoring; Parathyroid multiglandular disease

\section{Introduction}

The valid operative standard for the pHPT has been bilateral neck exploration (BNE) and the visualization of all parathyroid glands [1]. However, the increasing sensitivity of pre-operative localization methods such as 99Tc-sestamibi scanning and ultrasound in combination with intra-operative parathyroid hormone monitoring (ioPTH) assay have led to the use of minimally invasive procedures in parathyroid surgery. After first endoscopic parathyroidectomy, described by Gagner [1], in recent years, different minimally invasive techniques of parathyroidectomy have been described [2-6] and several authors have demonstrated that MIVAP is a safe procedure with additional advantages regarding cosmetic results and post-operative recovery [2-10].

We performed a retrospective study to analyse our decade experience in a single center from minimally invasive parathyroidectomy (MIP) in the management of our patients with $\mathrm{pHPT}$ and to demonstrate the feasibility of MIVAP and the utility of ioPTH determinations in detecting multiglandular disease.

pHPT is due to single gland disease in approximately $70-95 \%$ of cases, gland hyperplasia responsible for $15 \%$, double adenoma $4 \%$ or rarely caused by parathyroid carcinoma $[11,12]$.

The minimally invasive approaches increasingly replaced progressively the traditional BNE in the treatment of pHPT because of their significant advantages, especially in terms of post-operative pain, morbidity, operative time, and recovery. The use of this kind of approach has increased thanks to the improvement of localization techniques and to the use of the ioPTH monitoring [13-16].

George Irvin developed an immuno-chemiluminescence method, and the "quick" ioPTH assay became commercially available for intraoperative use [14-16].

Now-a-days, ioPTH is widely used in the majority of centers practicing parathyroid surgery. The most important advantage use of ioPTH is the possibilities to guide surgical decisions to confirm, intraoperatively, the complete removal of hyper-functioning parathyroid tissue or to guide the surgeon decide to perform BNE [17-21].

*Corresponding author: Dobrinja C, Division of General Surgery, Department of Medical, Surgical and Health Sciences, Cattinara Teaching Hospital, University of Trieste, Strada di Fiume, 34149, Trieste, Italy, Tel: 390403994152; Fax 390403994261; E-mail: ch_dobrinja@yahoo.it

Received May 24, 2017; Accepted June 24, 2017; Published June 26, 2017

Citation: Dobrinja C, Lanzaro A, Cubisino A, Stenner E, Ruscio M, et al. (2017) Effectiveness of Intraoperative Parathyroid Monitoring (IoPTH) During MinimallyInvasive Parathyroidectomy: A Decade Experience in a Single Center. J Mol Biomark Diagn 8: 349. doi: 10.4172/2155-9929.1000349

Copyright: (c) 2017 Dobrinja C, et al. This is an open-access article distributed under the terms of the Creative Commons Attribution License, which permits unrestricted use, distribution, and reproduction in any medium, provided the original author and source are credited. 
An intraoperative PTH drop of more than 50\% from the highest baseline value is highly precise (accuracy of $97 \%$ ) in predicting operative success [13-21]. The main reasons for an inappropriate ioPTH drop are multiglandular disease and microadenomas. The use of ioPTH is recommended especially for those patients undergoing minimally invasive operation with a single pre-operative localization study or two discordant pre-operative localization studies [22,23]

The main goal of our study was to analyse our results of minimally invasive parathyroidectomy. Furthermore, we aimed to confirm the usefulness of ioPTH when using minimally invasive techniques for treatment of sporadic pHTP.

\section{Materials and Methods}

From June 2005 to May 2017, we operated on 137 patients with pHPT. 60 patients $(43.8 \%)$ with pHPT were considered eligible for MIVAP. MIVAP by anterior approach, was proposed for patients with sporadic pHPT due to a single gland disease, with an adenoma smaller than $35 \mathrm{~mm}$ as demonstrated by pre-operative imaging, with localization of one unequivocally enlarged parathyroid gland on preoperative ultrasonography and concordant double-phase technetium Tc-99m (99m Tc) sestamibi scan, either alone or as 3-dimensional nuclear imaging fused with CT of the neck (single-photon emission computed tomography [SPECT]) pre-operatively, without secondary or recurrent hyperparathyroidism, no previous neck surgery, no previous radiation to the neck, without increased risk of multiglandular disease and the possibility of coexisting thyroid cancer. In all other cases (77 cases, 66.2\%) where we had discordance between ultrasonography and 99mTc-SestaMIBI scan, or a suspect of parathyroid malignancy, or for the surgeon choice and/or for the presence of associated neck diseases, we proposed MIP.

\section{ioPTH}

Quick parathyroid hormone immunochemiluminometric assay (Access Immunoassay System on Access-2 Beckman Coulter, UniCel ${ }^{\circ}$ DxI 800 Beckman Coulter - Fullerton, CA) was performed intraoperatively during the last 107 surgical procedures. Blood samples were collected in an EDTA tube from a peripheral vein by the anaesthesiologist at the designated times. Parathyroid hormone was measured at the induction of anaesthesia but before skin incision or any kind of neck manipulation (baseline level), at 5 and 10 minutes after removal of the suspected adenoma.

The ioPTH assays was performed in the main Laboratory next to the operating room. Samples were immediately transported to the nearby laboratory that was previously informed about the day and time of operation to allow enough time for pre-operative calibration and running of quality-control samples. Results were available after 15 minutes, the required incubation time and immediately communicated to the surgical team in the operating room.

Operative success, operative failure and recurrent hyperparathyroidism were defined with the same criteria described by Irvin et al. [13-16].

In the event intraoperative evidence of multiglandular disease is found (failure of background radiation or intraoperative PTH to appropriately decline), we evaluated the other 3 parathyroid glands.

\section{Recurrent laryngeal nerve integrity monitoring}

Intraoperative nerve monitoring (IONM) of the RLN can serve as an aid and adjunct to a minimally invasive procedure. While the RLN is not routinely identified during MIP, using IONM may help prevent a transient injury to the RLN [24]. IONM was utilised during the last 54 procedures.

Pre-operative informed consent was obtained from all patients. Procedures were performed by 3 different surgeons. Pre-operative and post-operative calcemia, phosphoremia and PTH levels were systematically evaluated in all patients. All patients underwent preoperative check of vocal cord function.

Regarding MIVAP, we used the operative technique first described by Paolo Miccoli [3], without carbon dioxide insufflation.

\section{Surgical technique: MIVAP}

The procedure, totally gasless, was performed trough a single 15-20 $\mathrm{mm}$ skin incision in the central neck, $1-2 \mathrm{~cm}$ above the sternal notch. The patient, under general anaesthesia, is placed in a supine position; the neck is in slight extension. Dissection was performed under endoscopic vision, using small conventional retractors and needlescopic $(2 \mathrm{~mm})$ reusable instruments. Video-assistance was obtained using a 30 degree $5 \mathrm{~mm}$ endoscope. The thyroid lobe was retracted medially and the adenoma was extracted after clipping its pedicle.

\section{Surgical technique: MIP}

The procedure, totally gasless, was identical as MIVAT technique but the dissection was performed under direct vision and the skin incision was a little bigger $(30-40 \mathrm{~mm})$.

Age, operative times, pain complaints during the first post-operative day, calcemia, PTH levels, length of hospital stay, pathologic findings, complications and cosmetic results, were retrospectively analyzed.

Follow-up evaluation included laryngoscopy to confirm vocal cord mobility in all patients three weeks after operation. In all patients, serum calcium levels, phoshoremia and PTH were systematically evaluated four hours after operation, in the first post-operative day, and 5 days after surgery. Hypocalcemia was defined as a serum calcium $<8.0$ $\mathrm{mg} / \mathrm{dL}$.

In case of post-operative serum calcium levels as low as $8.0 \mathrm{mg} / \mathrm{dL}$, patients were treated with substitutive therapy with Calcium carbonate tablets and with Dihydrotachysterol solution per os. The serum calcium level of hypocalcemic patients was rechecked every day during the recovery.

\section{Results}

Regarding the pre-operative imaging, in 60 patients $(43.8 \%)$ there was a localization of one unequivocally enlarged parathyroid gland on pre-operative ultrasonography and $99 \mathrm{mTc}$-SestaMIBI. These patients underwent MIVAP. In 64 patients (46.7\%) there was a localization of one enlarged parathyroid gland on pre-operative ultrasonography or on 99mTc-SestaMIBI (not both) therefore these patients underwent MIP.

In 13 cases (9.5\%) parathyroidectomy was performed after BNE. At the histological examination, 119 (86.9\%) patients had simple, single adenomatous pathology. We registered 5 (3.6\%) cases of atypical adenomas, $6(4.4 \%)$ cases of parathyroid multiglandular disease, and 4 histological exams of different nature (2.9\%). In 3 cases $(2.2 \%)$, a parathyroid carcinoma has been reported. Mean operative time was 87 minutes (range 20 to 218 minutes). Surgical cure of pHPT was achieved in all patients with calcemia levels normalization. Mean post-operative hospital stay was 2.1 days (range 1 to 6 days). 
Post-operative complications included 12 (8.75\%) transient asymptomatic post-operative hypocalcemia. No laryngeal nerve palsies, no definitive hypocalcemias (lasting more than 6 months after surgery), no persistent $\mathrm{pHPT}$ and no recurrent $\mathrm{pHPT}$ were observed. The ioPTH assay was used in 91 patients $(66.4 \%)$. Pre-operative median of calcium and PTH values (baseline) were $11.14 \mathrm{mg} / \mathrm{dL}$ (range: 4.00 - $18.80 \mathrm{mg} /$ $\mathrm{dL}$ ) and $326.4 \mathrm{pg} / \mathrm{mL}$ (range: $49.4-2414.7 \mathrm{pg} / \mathrm{mL}$ ) respectively. The median of PTH after 5 minutes was $88.98 \mathrm{pg} / \mathrm{mL}$ (range: 136 - 424 $\mathrm{pg} / \mathrm{mL}$ ), $59.32 \mathrm{pg} / \mathrm{mL}$ at 10 minutes (range: $12.8 \mathrm{pg} / \mathrm{mL}-214.6 \mathrm{pg} /$ $\mathrm{mL}$ ). Intraoperative PTH demonstrated a drop $>50 \%$ in respect to the baseline value in all patients, confirming successful parathyroidectomy.

\section{Discussion}

In the past, parathyroidectomy involved an open procedure with a neck incision not unlike that used for a thyroidectomy, and it required the inspection of all 4 parathyroid glands, with removal of the offending gland or glands $[1,2]$.

The main advantages of minimally invasive techniques over conventional parathyroidectomy include improved cosmetic results, less post-operative pain, less operative time, and reduced hospital stay. Larger series of clinicaland comparativestudieshavedemonstrated thatMIVAP is reproducible and safe, with an incidence of post-operative complications similar to that reported for the conventional approach [25-27]. Less post-operative pain might be related to the minimal incision and to the lower neck hyperextension reducing post-operative muscular pain.

Regarding the better cosmetic results of this technique, everyone agrees that a $2.0 \mathrm{~cm}$ scar is cosmetically better than that of traditional Kocher cervicotomy, which, in our department, is at least $6 \mathrm{~cm}$ to 10 cm long.

In our opinion, the availability of ioPTH assay is very important when performing MIP and other minimally invasive techniques to avoid surgical failures of overlooked multiple glandular disease [1317]. On the basis of this standard, we performed the ioPTH monitoring to confirm quantitatively when all hyperfunctioning parathyroid tissue has been removed. In fact, it avoids the need for BNE and identification of all parathyroid glands. This results in reduced operating time, limiting the overall costs of the procedure. Moreover, frozen section is not obligatory and does not give additional information when using qPTH assay. That results in a further reduction of the overall costs.

Results obtained with the use of quick dosage of IoPTH have been significant; no delay in the decrease of the levels of PTH and no qPTH assay false negative or false positive results were observed, but these results are still insufficient to be able to draw some definitive conclusions.

The indications for MIVAP are still limited. Nonetheless, in selected patients, it seems a valid alternative to the conventional surgery in patients with sporadic pHPT, with a small single adenoma unequivocally localized pre-operatively because of its significant advantages, especially in terms of cosmetic results, post-operative pain, and post-operative recovery.

Our results demonstrate that MIVAP, after adequate training and when selection criteria are strictly followed, is a feasible and safe surgical procedure, with good results, also in a General Surgery Department, from dedicated team, with a sufficient and specific activity volume.

The first aim of our study was to confirm the usefulness of ioPTH when using minimally invasive techniques for treatment of sporadic pHTP. The current immunochemiluminescence method used to detect
ioPTH was developed by Irvin and his collaborators [14-16]. Since that year this technique has been widely used to guide parathyroid surgery, helping - in association with the improvement in pre-operatory imaging techniques to perform a less invasive surgery [28,29]. Intraoperative PTH Assay during minimally invasive parathyroidectomy is considered helpful in the detection of double adenomas and may minimize the risk of recurrent surgery. In fact, it is demonstrated that when PTH did not drop $>50 \%$ from the first samples taken, the presence of a second hyperfunctioning parathyroid adenoma should be suspected. Our study confirms this trend, leading to a reduction of operative time (because it permits to avoid the frozen section (FS) examination of parathyroid gland, morbidity and post-operative pain, with a better aesthetic outcome [30,31]. From 2001 to 2016 ioPTH has been increasingly used associated to minimally invasive surgery (MIP/MIVAP), with a reduced use of conventional surgery technique and bilateral neck exploration. In our series, 70 patients (54.2\%) had FS with or without ioPTH monitoring: The execution of extemporary exam was associated with a longer operative time compared with ioPTH monitoring alone. Moreover, some studies show a superiority of ioPTH in detection of residual hyperfunctioning parathyroid tissue: FS can confirm the presence of hyperplastic parathyroid tissue, but can't give information about residual hyperfunctioning tissue in the neck $[23,25]$.

\section{Conclusion}

The use of ioPTH monitoring potentially offers increased sensitivity in detecting multiglandular disease can minimize the need and risk associated with recurrent operations, and may facilitate cost-effective minimally invasive surgery. Moreover, ioPTH monitoring is reliable, when pre-operatory imaging is available, to reinforce indication for a minimal-invasive approach and to reduce length of surgery even without FS exam.

\section{References}

1. Madkhali T, Alhefdhi A, Chen H, Elfenbein D (2016) Primary hyperparathyroidism. : Turk J Surg 32: 58-66.

2. Gagner M (1996) Endoscopc subtotal parathyroidectomy in patients with primary hyperparathyroidism. Br J Surg 83: 875.

3. Miccoli P, Pinchera A, Cecchini G, Conte M, Bendinelli C, et al. (1997) Minimally invasive, video-assisted parathyroid surgery for primary hyperparathyroidism. Endocrinol Invest 20: 429-430.

4. Henry JF, Defechereux T, Gramatica L, De Boissezon C (1999) Minimally invasive videoscopic parathyroid by lateral approach. Langebecks Arch Surg 384: 298-301.

5. Alshehri M, Mohamed HE, Moulthrop T, Kandil E (2017) Robotic thyroidectomy and parathyroidectomy: An initial experience with retroauricular approach. Head and Neck 5.

6. Sasanakietkul T, Jitpratoom P, Anuwong A (2016) Transoral endoscopic parathyroidectomy vestibular approach: A novel scarless parathyroid surgery. Surg Endosc 5: 1-9.

7. Bakkar S, Matteucci V, Corsini C, Pagliaro S, Miccoli P (2017) Less is more: Time to expand the indications for minimally invasive video-assisted parathyroidectomy. J Endocrinol Invest 3: 1-9.

8. Dobrinja C, Trevisan G, Liguori G (2009) Minimally invasive video-assisted parathyroidectomy. Initial experience in a general surgery department. J Endocrinol Invest 32: 130-133.

9. Miccoli P (2005) Parathyroid surgery: We only need a minimal surgical approach. J Endocrinol Invest 28: 570-573.

10. Dobrinja C, Silvestri M, De Manzini N (2012) Primary hyperparathyroidism in older people: Surgical treatment with minimally invasive approaches and outcome. Int J Endocrinol 2012: 539-542.

11. Reiher AE, Schaefer S, Chen H, Sippel RS (2012) Does the final intraoperative PTH level really have to fall into the normal range to signify cure? Ann Surg Oncol 19: 1862-1867. 
Citation: Dobrinja C, Lanzaro A, Cubisino A, Stenner E, Ruscio M, et al. (2017) Effectiveness of Intraoperative Parathyroid Monitoring (loPTH) During Minimally-Invasive Parathyroidectomy: A Decade Experience in a Single Center. J Mol Biomark Diagn 8: 349. doi: 10.4172/21559929.1000349

Page 4 of 4

12. Taubman ML, Goldfarb M, Lew JI (2011) Role of SPECT and SPECT/CT in the surgical treatment of primary hyperparathyroidism. Int J Mol Imaging 2011: 1-7.

13. Dobrinja C, Santandrea G, Giacca M, Stenner E, Ruscio M, et al. (2017) Effectiveness of intraoperative parathyroid monitoring (ioPTH) in predicting a multiglandular or malignant parathyroid disease. Int J Surg 41: S26-S33.

14. Irvin GL, Deriso GT (1994) A new, practical intraoperative parathyroid hormone assay. Am J Surg 168: 466-468.

15. Irvin GL, Molinari AS, Figueroa C, Carneiro DM (1999) Improved success rate in reoperative parathyroidectomy with intraoperative PTH assay. Ann Surg 229: 874-879.

16. Irvin GL, Solorzano CC, Carneiro DM (2004) Quick intraoperative parathyroid hormone assay: surgical adjunct to allow limited parathyroidectomy, improve success rate and predict outcome. World J Surg 28: 1287-1292.

17. Boggs JE, Irvin GL, Molinari AS, Deriso GT (1996) Intraoperative parathyroid hormone monitoring as an adjunct to parathyroidectomy. Surgery 120: 954958.

18. Westerdahl J, Bergenfelz A (2004) Sestamibi scan-directed parathyroid surgery: potentially high failure rate without measurement of intraoperative parathyroid hormone. World J Surg 28: 1132-1138.

19. Chen H, Pruhs Z, Starling JR, Mack E (2005) Intraoperative parathyroid hormone testing improves cure rates in patients undergoing minimally invasive parathyroidectomy. Surgery $138:$ 583-587.

20. Cayo AK, Sippel RS, Schaefer S, Chen H (2009) Utility of intraoperative PTH for primary hyperparathyroidism due to multigland disease. Ann Surg Oncol 16: $3450-3454$.

21. Irvin GL, Carneiro DM, Solorzano CC (2004) Progress in the operative management of sporadic primary hyperparathyroidism over 34 years. Ann Surg 239: 704-711.

22. Barczynski M, Konturek A, Cichon S, Hubalewska-Dydejczyk A, Golkowsk
F (2007) Intraoperative parathyroid hormone assay improves outcomes of minimally invasive parathyroidectomy mainly in patients with a presumed solitary parathyroid adenoma and missing concordance of preoperative imaging. Clin Endocrinol (Oxf) 8: 878-885.

23. Khan AA, Khatun Y, WalkerA, Jimeno J, Hubbard JG (2005) Role of intraoperative PTH monitoring and surgical approach in primary hyperparathyroidism. Ann Med Surg 4: 301-305.

24. Dionigi G, Boni L, Rausei S (2010) Minimally invasive videoassisted thyroidectomy and parathyroidectomy with intraoperative recurrent laryngeal nerve monitoring. Int J Otolaryngol 2010: 1-2.

25. Barczyński M, Cichoń S, KonturekA, Cichoń W (2006) Minimally invasive videoassisted parathyroidectomy versus open minimally invasive parathyroidectomy for a solitary parathyroid adenoma: a prospective, randomized, blinded trial World J Surg 30: 721-731.

26. Berti P, Materazzi G, Picone A, Miccoli P (2003) Limits and drawbacks of videoassisted parathyroidectomy. Br J Surg 90: 743-747.

27. Miccoli P, Berti P, Materazzi G, Donatini G (2003) Minimally invasive video assisted parathyroidectomy (MIVAP). Eur J Surg Oncol 29: 188-190.

28. Shapey IM, Jabbar S, Khan Z, Nicholson JE, Watson RJ (2017) Scan-directed mini-incision focused parathyroidectomy: how accurate is accurate enough? Ann R Coll Surg Engl 99: 123-128.

29. Wilhelm SM, Wang TS, Ruan DT, Lee JA, Asa SL, et al. (2016) The American Association of Endocrine Surgeons: Guidelines for definitive management of primary hyperparathyroidism. JAMA Surg 151: 959-968.

30. Dobrinja C, Stenner E, Trevisan G, Micheli W, Biasioli B, et al. (2009) Minimally invasive video-assisted parathyroidectomy and intraoperative parathyroid hormone monitoring. Am J Surg 31: 319-321.

31. Badii B, Staderini F, Foppa C, Tofani L, Skalamera I, et al. (2017) Costbenefit analysis of the intraoperative parathyroid hormone assay in primary hyperparathyroidism. Head and Neck 39: 241-246. 